Bojana Kovačević Petrović ${ }^{1}$ Universidad de Novi Sad Facultad de Filosofía y Letras
UDK: 929:[811.133.4+821.133.4 Soldatić D.

https://doi.org/10.18485/imp.2017.ch.20

\title{
DALIBOR SOLDATIĆ Y LA LITERATURA HISPANOAMERICANA EN SERBIA
}

A través de varias entrevistas y auténticos testimonios hechos a propósito de esta investigación, el presente artículo pretende mostrar la contribución del Dr. Dalibor Soldatić al hispanismo de Yugoslavia y de Serbia. Considerando su trayectoria de profesor de la Facultad de Filología de la Universidad de Belgrado y el Jefe del Departamento de Estudios Ibéricos de la misma institución, sus artículos científicos publicados en diversas revistas académicas, su traducción de una veintena de libros, su experiencia como vicedecano de Enseñanza/Ciencia y Relaciones Internacionales de la Facultad de Filología (en dos mandatos) y el Vicerrector de Relaciones Internacionales de la Universidad de Belgrado, tanto como su apoyo a los estudiantes y a los colegas más jóvenes, en el presente trabajo pondremos acento en el impacto de Dr. Soldatić en la recepción de la literatura hispanoamericana en Serbia y en su pensamiento crítico sobre los temas hispánicos. Una parte de nuestro artículo estará dedicada a la enseñanza de la literatura española e hispanoamericana en los 45 años del Hispanismo en esta región y las visiones del futuro de los estudios de filología hispánica del propio Soldatić, galardonado por su trabajo con la Orden Isabel la Católica que otorga el Gobierno de España y con La Orden Mexicana del Águila Azteca.

Palabras clave: Dalibor Soldatić, hispanismo en Yugoslavia, hispanismo en Serbia, traducción del español al serbio, Facultad de Filología de la Universidad de Belgrado, recepción de la literatura hispanoamericana en Serbia, enseñanza de la literatura española e hispanoamericana.

\section{Introducción}

Dalibor Soldatić es considerado uno de los hispanistas más importantes de Yugoslavia y Serbia y uno de los pioneros del hispanismo en estos países. Nacido en Argentina en 1947 y educado en Split (hoy Croa- 
cia), Belgrado y México, se licenció por la Universidad Autónoma de México (UNAM) aprobando el tercero y el cuarto curso en un año e hizo su tesis de maestría en la misma universidad, titulada La nostalgia en la obra poética de Rafael Alberti, en solo un año y con las mejores notas. Después de los años mexicanos, Soldatić empezó a trabajar en la Facultad de Filología de Belgrado, como asistente de la primera profesora de filología hispánica, la Dra. Ljiljana Pavlović Samurović. Las investigaciones de los materiales literarios necesarios para su futura tesis las hizo en París, el centro mundial de la literatura hispanoamericana en esa época. De vuelta a Belgrado, se doctoró en el año 1984 en la Teoría de novela de Mario Vargas Llosa como et primer hispanista en la historia de la Facultad de Filología de Belgrado. Aparte de su trayectoria universitaria, Dalibor Soldatić ha sido Asesor de Prensa de la Embajada de la República Federativa Socialista de Yugoslava en Roma (1990-1992) y Encargado de Negocios de la República Federal de Yugoslavia (1992-1993) en la capital italiana. Por muchos años ha sido Jefe del Departamento de Estudios Ibéricos, Vicedecano de Relaciones Internacionales y Ciencia (2006-2010), y entre 2012 y 2015 el Vicerrector de Relaciones Internacionales de la Universidad de Belgrado. Asimismo, Soldatić es el punto crucial del desarrollo del hispanismo en Belgrado, Kragujevac y Novi Sad, autor de decenas de traducciones de libros españoles e hispanoamericanos y artículos científicos sobre temas hispanos, gran soporte de la Asociación de Hispanistas serbios y de la Red de hispanistas de Europa Central y laureado de la Orden Isabel la Católica que otorga el Gobierno de España y La Orden Mexicana del Águila Azteca.

Teniendo todo esto en cuenta, en el presente artículo intentaremos mostrar todos los aspectos de la contribución de Dalibor Soldatić al hispanismo en esta región, a través de los libros y textos que ha escrito y traducido, pero sobre todo a través los testimonios de sus colegas y colaboradores, basados en varias entrevistas que hemos hecho a ellos y a él mismo.

\section{Los comienzos del hispanismo en Yugoslavia y su desarrollo en Serbia}

La enseñanza de la lengua española empezó a impartirse en Belgrado a finales del año 1951, como asignatura optativa que ofertaba el 
Departamento de Lenguas y Literaturas Románicas de la Facultad de Filosofía. En 1960 ese departamento se integró en la Facultad de Filología de la Universidad de Belgrado y en 1971 se fundó el primer Grupo de Lengua y Literatura Española de la antigua Yugoslavia, que pasó a formar parte del Departamento de Lenguas y Literaturas Románicas². Una de las primeras estudiantes fue Dragana Bajić, a quien le entrevistamos a propósito de nuestro artículo:

Empecé a estudiar Lengua y Literatura Española en 1973, como tercera promoción de la joven cátedra establecida en 1971, dentro del Departamento de Lenguas Románicas que abarcaba los estudios de francés, español y rumano. En aquel entonces había muy pocos docentes: la Prof. Dra. Lj. Pavlović-Samurović y su ayudante Dalibor Soldatić, que unos años después se doctoró. Los dos impartían clases tanto de lengua como de literatura. Para las clases prácticas estaban encargados como lectores Silvia Izquierdo-Todorović y Juan Octavio Prenz, que muy pronto se marchó de la Facultad de Filología. Al terminar los estudios seguí con el postgrado y empecé a trabajar junto con Jelena Rajić como ayudante de profesor en las asignaturas de lengua, impartiendo clases prácticas de fonética, morfología, sintaxis, gramática histórica, análisis contrastivo y traducción de textos clásicos y modernos. (Bajić 2016)

A nuestra pregunta qué trajo de América Latina después de sus estudios mexicanos, Soldatić respondió que "sobre todo había traído la pasión hacia la literatura hispanoamericana" (Soldatić 2015). En la segunda entrevista que le hicimos a Dalibor Soldatić a propósito de este artículo, él nos comentó que "tanto la profesora Samurović como yo queríamos desarrollar la cátedra y nuestra idea siempre fue igualar el estudio de la lengua y de la literatura" (Soldatić 2016). Así en los años setenta se introdujo la asignatura Literatura Hispanoamericana "que se estudiaba como materia de dos semestres. Cuando vimos que el material era dema-

2 La enseñanza de la lengua española empezó a impartirse en Belgrado a finales del año 1951 como una asignatura optativa del Departamento de Lenguas y Literaturas Románicas de la Facultad de Filosofía. Ese departamento se integró en la Facultad de Filología en 1960. Véase: http://old.fil.bg.ac.rs/katedre/spanski/espanol/istorijat.htm. 
siado voluminoso, lo dividimos en dos años" (idem). Con la introducción de la nueva asignatura, se amplió el programa de los estudios básicos de literatura, y en el programa de enseñanza de lengua se incluyó la investigación de las características específicas del español en los territorios de la América Hispánica. En esa época el futuro Departamento cambió de su nombre a Grupo de Lengua Española y Literaturas Hispánicas, aunque tras introducir los estudios de las lenguas portuguesa y catalana, en el año 2000 el Grupo pasó a denominarse Cátedra de Estudios Ibéricos.

En 1996, como parte de una colaboración recíproca con la Universidad de Granada, se creó el lectorado de lengua serbia en la Facultad de Filosofía de dicha ciudad: "Granada fue una casualidad. Me llamaron de la Embajada de España diciéndome que había un profesor de Granada, profesor de ruso Rafael Guzmán Tirado, que quería introducir, además del polaco y checo, también la lengua serbo-croata" (Soldatić 2016). El consejero le dijo a Dalibor Soldatić que podía ponerse en contacto con él si le parecía buena la idea, y el Decano y él decidieron mandar un lector allí, se fueron a Granada y según nos ha contado Soldatić "nos pusimos de acuerdo y les llevamos muchísimos libros, 200 kilos. Ese lectorado desgraciadamente se cerró debido a cortes de presupuesto y falta de interés de los alumnos. La culpa es nuestra también, que no hemos sabido dar publicidad, anunciarlo".

Entre tanto, en la Facultad de Filosofía de la Universidad de Novi Sad ${ }^{3}$ en 1997 empezó a impartirse la lengua española dentro del Departamento de Lenguas y Literaturas Romances gracias al profesor Milorad Arsenijević. El nuevo programa del Lectorado, dirigido desde 2004 por Dra. Ksenija Šulović, hoy día cuenta con cinco docentes y planes de ampliación, abarca la Lengua Española, la Cultura Hispánica y varias asignaturas de maestría.

En el año 2006 se generó la idea de ampliar oferta de idiomas en la Facultad de Filología y Letras de Kragujevac y abrir un Departamento de estudios hispánicos, que "es puro mérito del rector de aquella época y yo al principio ofrecí mucha resistencia cuando me nombraron el jefe del Departamento de Español" (Soldatić 2016). Hoy hay muchos festivales y mucho movimiento allí, pero en ese momento Kragujevac era una ciudad provincial sin un solo hispanista formado. "Menos mal, el rector insistió y ahora tengo que decir 
que mi idea era equivocada", nos comentó Soldatić en mayo de 2016. La existencia de la Cátedra de Estudios Hispánicos ${ }^{4}$ ha permitido que un gran número de alumnos estudiaran letras hispánicas que de otro modo no hubieran podido. "Kragujevac atrajo la región de Šumadija, Despotovac, Milanovac, etc. Por otra parte, el equipo que se constituyó allí, encabezado por Anđelka Pejović, fue un equipo joven con mucha libertad de movimiento y para experimentar, así que se hizo una cátedra dinámica" (Soldatić 2016).

\section{El boom de la literatura hispanoamericana en Serbia}

Uno de los años cruciales de la difusión de la literatura hispanoamericana en Yugoslavia, fue 1969, cuando la casa editorial Zora de Zagreb publicó la traducción de la novela La muerte de Artemio Cruz de Carlos Fuentes (hecha por Edita Marijanović), con el epílogo de Dalibor Soldatić como un texto crítico sobre la vida y la obra de uno de los futuros escritores más grandes de América Latina. Solo siete años tras su publicación en español, los lectores serbios y croatas tenían en su idioma la obra que representaba la afirmación definitiva de Carlos Fuentes y el cambio crucial de su creación literaria (Soldatić 1969: 252) ${ }^{5}$.

Antes de la publicación de la revista que produciría el boom de la literatura hispanoamericana en Serbia, Soldatic escribió en 1976 el prólogo para una antología de la poesía española contemporánea, editada y traducida por Silvia Monrós Stojaković y publicada por Bagdala de Kruševac. Un año después, el artículo basado su tesis de maestría "Izgubljeni raj Rafaela Albertija - Sobre los ángeles" (El paraíso perdido de Rafael Alberti) fue publicado en Sarajevo ${ }^{6}$ como el primer texto de Alberti en Yugoslavia ${ }^{7}$.

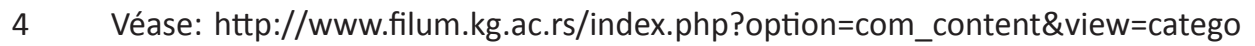
ry\&layout=blog\&id=248\&ltemid=294

5 Por cierto, éste no fue el primer artículo publicado por Soldatić. Dos años antes, en México apareció la primera reseña escrita por él: Aurora M. Ocampo de Gómez y Ernesto Prado Velázquez., "Diccionario de escritores mexicanos» In: La palabra y el hombre. Xalapa, Ver. México. 1967. №. 44 . pp. 844-846.

6 Izraz. Sarajevo. 1977. XXI. №. 8-9. pp. 1157-1171.

7 Un año más tarde Jordan Jelić traducirá una veintena de poemas de Alberti para la revista Polja, Año 24, No. 230 (abril 1978) que en 1981 publicará bajo el título 
Dalibor Soldatić y otro destacado hispanista serbio, Branko Anđić, colaboraron en la creación de la primera Antología del cuento hispanoamericano, que Anđić había hecho para la editorial Srpska Književna Zadruga y que fue publicada en 1980. Por aquel entonces, algunos escritores y traductores empezaron a darse cuenta de varios libros aislados hispanoamericanos que surgieron en las librerías, en general traducidos de otros idiomas, pero hechos con entusiasmo. En la entrevista que le hicimos a Branko Anđić a propósito de la investigación científica, él se acordó de muchas cosas de esa época: "Soldatić y yo estuvimos muy conscientes de la importancia del momento y de la cantidad de libros por traducir - porque en ese momento el hispanismo era, por decirlo así, inexistente en Serbia" (Anđić 2015). Luego empezaron a buscar a la gente que no necesariamente debía ser del ámbito hispánico, pero que hablaba español muy bien y que tenía un amplio conocimiento literario. Milan Komnenić, quien fue editor en la casa editorial Prosveta, estaba también muy interesado en la literatura hispanoamericana, que leía en "francés e italiano, porque sabía que en el mundo ya había empezado el boom. Nosotros tuvimos un retraso de más o menos diez años, que desde la perspectiva actual es muy poco, porque hoy nuestro retraso es mucho más grande“ (Anđić 2015). Komnenić decidió arriesgar con la publicación de Aura de Carlos Fuentes ${ }^{8}$, que resultó un enorme éxito: "Krajem sedamdesetih godina, u biblioteci Erotikon naišao sam na roman koji i danas smatram jedni od vrhunaca ne samo hispanoameričke već i svetske književnosti, a to je Fuentesova Aura. Bio sam fasciniran. Kao mlad čitalac i pisac postavio sam sebi pitanje: zar se i ovako može pisati?"9 (Damjanov, 2016). En esas circunstancias, Soldatić y Anđić empezaron a reflexionar sobre la posibilidad de "gestionar una serie de libros para presentar por lo menos las novelas latinoamericanas más importantes, pero sabíamos muy bien que nos faltaba el contexto, así que decidi-

Mornar na kopnu i druge pjesme (Marinero en la tierra y otros poemas) en Titograd (hoy Podgorica).

8 Primera edición: Prosveta, Belgrado (Biblioteka Erotikon), 1978. Traducción Branko Anđić.

9 "A finales de los años setenta, en la biblioteca Erotikon me encontré con una novela que todavía hoy en día considero una de las mejores, no solo de la literatura latinoamericana, sino también de la literatura universal : Aura de C. Fuentes. Me fascinó. Como joven lector y escritor me hice a mí mismo la pregunta: ¿es posible escribir también de esa manera?" (Trad. aut.) 
mos empezar por otro lado." (Anđić 2015). En esa época grandes profesores y críticos latinoamericanos -como Ángel Rama o Julio Ortega- ya habían publicado varios ensayos sobre el boom y teniendo eso en cuenta, Soldatić y Anđić decidieron editar una revista de textos críticos y fragmentos de varias obras, que daría el contexto para la futura edición de las grandes novelas hispanoamericanas. Como en Belgrado en los años setenta había por lo menos una decena de revistas literarias de gran calidad, y los editores jefes eran personas que entendían de literatura, su idea fue inmediatamente aceptada por el editor Jovica Aćin. Tras buscar los materiales, juntarlos y escoger a los escritores (para eso necesitaron más de seis meses), Soldatić y Anđić publicaron el número especial de la revista mensual de teoría, crítica y poesía Delo ${ }^{10}$, “especialmente diseñado, que recibió apodo 'el Delo verde' y resultó que ese número lo leería mucha más gente de lo esperado" (Anđić 2015). Aparte de varios textos críticos $^{11}$ traducidos de español y cuentos y fragmentos de los libros más significantes del pre-boom y boom ${ }^{12}$, la revista contiene una introducción de los editores sobre el "Panorama de la nueva literatura hispanoamericana" donde se explica su idea y el título del número especial, que les pareció adecuado no solo por el vínculo con el cuento homónimo de Jorge Luis Borges, sino "zbog činilaca mešanja i spoja najrazličitijih etničkih i kulturnih uticaja koji često istovremeno dovode do haosa i monumentalnosti, do košmara i velelepnosti“13 (Anđić y Soldatić 1978: 3). En su texto informativo, crítico y desde luego pionero sobre "La novela hispanoamericana hoy", después de un breve recorrido por los precursores del boom y la situación actual, Soldatić en la revista Delo afirma que en Yugoslavia también ha empezado

10 «Vavilonska biblioteka. Nova hispanoamerička književnost». (La biblioteca de Babel. Nueva literatura hispanoamericana). Delo, número especial (Editores Dalibor Soldatić y Branko Anđić). Belgrado. XXIV. 1978. №. 8-9. Agosto-septiembre. 258 pp.

11 De José Luis Martínez, Jaime Valdivieso, Mario Benedetti, Octavio Paz, Carlos Rincón, entre otros.

12 Toda la elite de la literatura hispanoamericana: Agustín Yáñez, Juan Rulfo, Alejo Carpentier, Carlos Fuentes, Augusto Roa Bastos, Gabriel García Márquez, Jorge Luis Borges, Julio Cortázar, Ernesto Sábato, Manuel Puig, Mario Vargas Llosa, Alfredo Bryce Echenique, José Donoso y Mario Benedetti.

13 "por los elementos de mezcla y la diversidad de influencias étnicas y culturales que a menudo conducen al mismo tiempo al caos y a la monumentalidad, a la pesadilla y al esplendor". (Trad. aut.) 
el boom de la literatura hispanoamericana, pero que la novela de ese continente sigue prácticamente desconocida, y que "konstatacija da je to uslovljeno nedostatkom hispanističke tradicije kod nas, da objavljivanje tih dela nije bilo propraćeno ni prigodnim prikazima, člancima a još manje ozbiljnim studijama, verovatno nije dovoljna da opravda takvo stanje, ali sigurno deluje kao ilustracija“14 (Soldatić 1978: 41). Por otro lado, varias décadas después, Soldatić comenta que el así llamado "boom de la literatura hispanoamericana es el fruto de un largo procedimiento de maduración de cierto tipo de expresión literaria, con una buena base en las obras de Macedonio Fernández, Juan Rulfo, Ernesto Sábato, Alejo Carpentier, Agustín Yáñez etc." (Soldatić 2015) y explica que el fortalecimiento de la clase media, aumento del número de estudiantes, y por consiguiente los escritores potenciales, seguido por el desarrollo del mundo editorial, resultó con el gran interés en la nueva novela hispanoamericana, y luego con su internacionalización. Según las palabras de Soldatić, en ese momento el número especial de Delo no tuvo el impacto tan grande como parece hoy: "tuvimos que pagar por el gran entusiasmo y el deseo de publicarlo, porque había unas traducciones falladas y muchas erratas" (Soldatić 2015).

Tras la publicación del "Delo verde" los editores abrieron sus casas editoriales para los escritores hispanoamericanos ${ }^{15}$ y los años ochenta fueron muy fructíferos para el hispanismo en Serbia. En 1980 apareció la

14 "La opinión que eso es causado por la falta de la tradición hispana en nuestra región, que la publicación de esas obras no ha sido acompañada de reseñas y artículos adecuados, ni mucho menos con unos estudios serios, probablemente no sea suficiente para justificar tal situación, pero ciertamente puede ser una ilustración de ella." (Trad. aut.)

15 Hasta entonces, había varias traducciones de la literatura hispanoamericana: Pedro Páramo de Juan Rulfo (1966), Las armas secretas de Julio Cortázar (1969 y 1978), ambas traducidas por Radoje Tatić, y hasta Cien años de soledad de García Márquez (1973), pero a pesar de eso no hubo mucho interés. Incluso, según el testimonio de Branko Anđić, cuando Jasna Mimica tradujo la novela Cien años de soledad las primeras cinco casas editoriales rechazaron publicarla, aunque el libro ya tenía éxito mundial. Cuando BIGZ por fin decidió publicarlo -no por el libro sino por la línea privada- resultó que un año después no había persona educada en Belgrado que no hubiera leído esa novela (Anđić, 2015). Otra razón fue una encuesta que el periódico Política le hizo al profesor de literatura general, Vladeta Košutić, quien declaró que para él el acontecimiento cultural del año fue la publicación de Cien años de soledad y de repente todo el mundo empezó a leerlo (Soldatić, 2015). 
mencionada primera Antología del cuento hispanoamericano contemporáneo (seleccionada y editada por Branko Anđić), con un bien estructurado prólogo de Dalibor Soldatić donde se explica el desarrollo histórico del cuento en América Latina y recorren los movimientos y las corrientes cruciales: romanticismo, realismo, naturalismo, modernismo, regionalismo y "nueva literatura", y confirma "la madurez y la originalidad del cuento hispanoamericano como género literario" (Soldatić 1980: XXVIII).

Puesto que en los años ochenta la situación fue mucho mejor entre los editores en cuanto a la literatura hispanoamericana, a Soldatić y a Anđić se les ocurrió preguntar a varias casas editoriales si querrían colaborar en la publicación de una serie de novelas hispanoamericanas. Los primeros editores jefes que aceptaron su proyecto fueron Vuk Krnjević y Milan Komnenić, y los dos hispanistas contaron además con el apoyo de Svetlana Velmar, que en aquel entones era editora de la Prosveta. Ellos "empezaron a llamar por teléfono a otras casas editoriales -NOLIT, Partizanska knjiga, Narodna knjiga, Svjetlost de Sarajevo- para preguntar si querían participar en el proyecto y llegaron a un acuerdo con todos en nada más dos días" (Anđić 2015). Luego empezó otro tipo de problema: cómo escoger diez libros más importantes de la literatura hispanoamericana, a quién excluir, cómo traducir, por ejemplo Paradiso de José Lezama Lima o Tres tristes tigres de Guillermo Cabrera Infante, y tras elegir los escritores y los traductores - cómo decidir entre varios libros suyos ( $L a$ muerte de Artemio Cruz o Terra Nostra, Túnel o Sobre héroes y tumbas, por ejemplo, como afirma Anđić), pero al final optaron por los libros que no se habían publicado antes en Yugoslavia ${ }^{16}$. Veinte años después de traducir Terra Nostra, Marica Josimčević comentó que había aceptado el trabajo prácticamente sin pensar, tras leer quince páginas de la novela:

16 La primera serie (1984) contenía las traducciones de las siguientes novelas : El astillero de Juan Carlos Onetti (tr. Marija Dimitrijević), Sobre héroes y tumbas de Ernesto Sábato (tr. Slavica Kojić), Yo, el Supremo de Augusto Roa Bastos (tr. Jasna Bunjevac Sotelo), Rayuela de Julio Cortázar (tr. Silvia Monros Stojaković) y Conversación en la Catedral de Mario Vargas Llosa (tr. Jasna Bunjevac Sotelo); y la segunda (1985): El Siglo de las Luces de Alejo Carpentier (tr. Danica Moreno), Cien años de soledad de Gabriel García Márquez (tr. Jasna Mimica Popović), Terra Nostra de Carlos Fuentes (tr. Marica Josimčević), El beso de la mujer araña de Manuel Puig (tr. Dubravka Popović-Srdanović) y El obsceno pájaro de la noche de José Donoso (tr. Branko Anđić). 
Toj erudicijskoj prašumi, tom kolosu savremene književnosti poklonila sam četiri godine svog života. Ne bih umela da kažem zašto sam gotovo trenutno prihvatila prevođenje, sem da me je još od prvih redova knjige ščepalo neko tajanstvo, razlilo mi se po telu, opilo me. Izići iz rada na toj knjizi značilo je za mene izići iz čuda stvaranja koje se očitovalo u svakoj rečenici, odeljku, poglavlju. ${ }^{17}$ (Josimčević 2000: 103)

Aparte de contratar a los jóvenes hispanistas -ex estudiantes de Soldatić- para hacer las traducciones, los editores decidieron acompañar los libros con amplios epílogos. En este artículo destacaremos dos, escritos por Dalibor Soldatić: "La obra literaria de Mario Vargas Llosa"18, postfacio de la versión serbia de la Conversación en la Catedral (1984) y "Carlos Fuentes y el mito de la creación literaria" publicado en el segundo libro de Terra Nostra. El primer texto abarca la vida y la carrera de Vargas Llosa desde su primera obra hasta La guerra del fin del mundo, con acento en la estética, la estructura y el procedimiento narrativo de Vargas Llosa, y el segundo, tras el recorrido por las obras publicadas antes de Terra Nostra, ofrece una crítica detallada y profunda de la novela capital de Carlos Fuentes, sus temas, su estructura, la(s) realidad(es) que trata, los personajes cruciales y el tema eterno de la identidad hispanoamericana.

Como habían mencionado tanto Anđić como Soldatić, la publicación de Rayuela abrió paso a la futura traducción de todos los cuentos de Julio Cortázar, hecha por Aleksandra Mančić entre 1987 y 2012, que causaría un verdadero culto de Cortázar en Serbia.

Entre varias anécdotas que acompañaron el desempeño de las mencionadas series de libros, queremos mencionar dos: Branko Anđić se gastó

17 A esa selva tropical de erudición, a ese coloso de la literatura contemporánea, le regalé cuatro años de mi vida. No sé cómo explicar por qué acepté traducirlo casi al instante, solo puedo decir que algún misterio me agarró desde las primeras líneas de esa novela, me cayó sobre el cuerpo, me embriagó. Salir de ese libro para mí significaba salir de la maravilla de creación, que se reflejaba en cada frase, en cada fragmento, en cada capítulo. (Tr. aut.)

18 Tres décadas después Soldatić participará en el Simposio dedicado a Mario Vargas Llosa en Montenegro, dará una ponencia sobre su obra en el Teatro Nacional Serbio en Novi Sad antes de la conversación con el Nobel peruano, y tendrá el honor de otorgarle en Premio "Milovan Vidakovic" de ProseFest. 
todo el honorario en las llamadas telefónicas con José Donoso mientras traducía El obsceno pájaro de la noche y Ernesto Sábato aceptó que le pagaran los derechos de autor por Los héroes y tumbas con un cuadro de un pintor serbio, porque las editoriales no disponían de moneda extranjera.

\section{Libros y artículos publicados}

Aunque en nuestro artículo queremos poner acento en el desempeño de Dalibor Soldatić en el impacto de la literatura hispanoamericana en Serbia, consideramos importante mencionar su significativa contribución al libro La literatura española I (edad media y renacimiento) / Španska književnost I (srednji vek i renesansa), que escribió junto con Ljiljana Pavlović Samurović ${ }^{19}$ en 1985, la publicación con Soldatić como co-editor: Baladas y leyendas populares en España y Yugoslavia (Actas del Coloquio Internacional llevado a cabo el 5 de abril de 2002), con J. Nikolić y C. Díez Plaza y actas del Congreso Language, Literature, Culture, Identity. Proceedings of the International Conference, Belgrade, 2009, editadas con Slobodan Grubačić.

En 2002 Dalibor Soldatić publicó de momento la única monografía serbia dedicada al boom de la literatura hispanoamericana y sus precursores: Las contribuciones a la teoría de la nueva novela hispanoamericana (Prilozi za teoriju novog hispanoameričkog romana), abriendo nuevos caminos de la recepción de los libros publicados en ese continente y el género novelesco desarrollado por los escritores españoles e hispanoamericanos: "Autor se u ovoj studiji javlja kao višestruki istraživač: kao lingvista, kao istraživač iz oblasti nauke o književnosti, kao sociolingvista i kao istoričar"20 (Đurić 2005: 126). Entre varios temas que abarca su monografía figuran: la realidad objetiva, la realidad de una obra literaria, el realismo mágico, lo real maravilloso, la literatura fantástica, la novela total,

19 Ljiljana Pavlović Samurović y Dalibor Soldatić: Španska književnost I.(Literatura española I) Sarajevo. Svjetlost. 1985. 354 pp.

20 El autor de este estudio se presenta como un investigador múltiple: como lingüista, como investigador en el campo de los estudios literarios, como sociolingüista y como historiador. (Trad. aut.) 
etc. En otras palabras, "este libro (299 páginas, $24 \mathrm{~cm}$ ) es resultado de los muchos años que el autor lleva investigando y estudiando la literatura de la América Latina, especialmente la nueva novela hispanoamericana, que se convirtió en el campo de su más estrecha especialidad profesional" (Dickov 2014: 96). Reflexionando sobre la teoría de la novela en el mundo hispanohablante, la actitud frente a la realidad como cuestión clave de la novela hispanoamericana y el pensamiento teórico de Ernesto Sábato, Alejo Carpentier, Carlos Fuentes y Mario Vargas Llosa, Soldatić llegó a varias conclusiones sobre el estatus contemporáneo de la novela, al cual responden, según él:

Las poéticas individuales y las situaciones de un debate permanente: el diálogo se sostiene tanto dentro de la novela como entre novelas de orientación distinta, entre la novela y los textos con otra finalidad, entre la novela y la política, la novela y la filosofía, la novela y la ciencia, el diálogo que representa ahora una conversación entre participantes en pie de igualdad. (Soldatić 2002: 287)

Otro libro suyo que queremos destacar abarca un enorme número de temas hispanos, desde el desarrollo del hispanismo en el mundo y dentro de Serbia, pasando por las reflexiones sobre el idioma español como uno de los más significativos del planeta, ofreciendo datos sobre la historia de España y América Latina y los medios de comunicación en los dos continentes hispanohablantes, mencionando las instituciones más importantes y los premios literarios españoles e hispanoamericanos. La monografía Svet hispanistike - Uvod u studije (El mundo del hispanismo: Introducción a los estudios) Soldatić la escribió con Željko Donić, quien trató las artes plásticas de España y América Latina, cinematografía, música y cultura popular de los dos continentes.

Entre varios artículos y una cantidad de reseñas que Dalibor Soldatić ha publicado en revistas nacionales e internacionales, en este artículo queremos destacar los textos relacionados con la literatura hispanoamericana: "El ensayo hispanoamericano" (Treći program Radio Sarajeva. Sarajevo. 1979-1980. Núm. 27-28., pp. 291-299), "Tres tipos de relación con la realidad en la prosa hispanoamericana. Realismo mágico, lo real maravilloso y la literatura fantástica" (Treći program Radio Sarajeva. 1986. 
№. 52. pp. 82-133), “Diccionario de literatura hispanoamericana de Ljiljana Pavlović Samurović" (Rassegna Iberistica. Roma. 1997. №. 60. pp. 60-65), "Novela hispanoamericana e historia" (América Latina en el siglo XX, Belgrado, Asociación Yugoslava de Latinoamericanistas, 1999, pp. 355369), "Una literatura de confrontación de lo verídico con lo falso" (Temas literarios mexicanos, ed. Moma Dimić, Asociación de escritores de Serbia, Belgrado, 2000, pp. 11-22), "Pedro Páramo: historia de una búsqueda frustrada"21 (Beogradski književni časopis. Hiperboreja, Belgrado, Año II., núm. 2/15, marzo 2006, pp. 215-219), “José Martí escritor, revolucionario" (Cuba en el umbral del tercer milenio, Interpress y Asociación Yugoslava de Latinoamericanistas, Belgrado, 2006, pp. 32-38, "La conquista de América Latina y la administración colonial como una clave para acceder a la literatura hispanoamericana" (Filološki pregled, Beograd, XXXIV2007.2, pp. 55-69), "Novela hispanoamericana actual" (Tokovi u savremenoj romanistici, Filološki fakultet y Asociación para la Cooperación Cultural Serbia-Francia, Belgrado, 2008, pp.47-56), "Las literaturas hispánicas en Serbia" (Colindancias, Revista de la Red Regional de Hispanistas de Hungría, Rumania y Serbia, Timisoara, núm. 2010, pp. 21-28) y Novela mexicana e historia (Nasleđe, Kragujevac,VIII, №.18, 2011, pp. 289-299) en el número especial titulado Novela española e hispanoamericana - lengua, ideología, discurso, historia, poética, (editores Dalibor Soldatić, Anđelka Pejović, Jasna Stojanović, Vladimir Karanović y Mirjana Sekulić, 427 p.)

También hace falta mencionar la reseña del Libro sobre Cervantes (2a. edición aumentada) de Ljiljana Pavlović Samurović (Espéculo. Revista de estudios literarios. Universidad Complutense de Madrid. 9/11/2005) y de la nueva traducción de Don Quijote hecha por Aleksandra Mančić en 2005 y de su Diario de traducción de la novela de Cervantes Vetrenjače na jezik, que Soldatić ha publicado en Beogradski književni časopis No. 2

21 En las reflexiones sobre la novela de Juan Rulfo, base crucial del futuro boom, Soldatić apunta: "Iz tog novog hispanoameričkog romana izdigla se i nova slika latinoameričkog kontinenta. To više nije kontinent izneverenih revolucija, državnih udara, gladi i nerazvijenosti, već ikontinent koji traži sopstvene, originalne puteve prosperiteta" (2006, 217). ("De la nueva novela hispanoamericana se ha gestionado una nueva imagen del continente latinoamericano. Ya no se trata de un continente de revoluciones fracasadas, golpes de estado, hambrunas y subdesarrollo, sino del continente en la búsqueda de sus propios caminos originales de la prosperidad." Tr. aut.) 
(primavera 2006) y necrología dedicada a la fallecida pionera del hispanismo serbio Dra. Ljiljana Pavlović Samurović (Zbornik Matice srpske za književnost i jezik, libro LIV, tomo 2/2006, pp. 257-259)

\section{Traducciones}

Durante más de veinte años Dalibor Soldatić había trabajado como intérprete simultáneo, no solo del español al serbio sino también del serbio, francés e inglés al español. "En esa época los salarios del profesorado eran bastante pequeños y si uno quería tener una vida buena tenía que hacer algo aparte, algo adicional" (Soldatić 2016). Soldatić se estrenó como intérprete por pura casualidad; su primera interpretación simultánea fue un discurso inaugural del presidente Tito en un Congreso del Partido Comunista:

Había dos intérpretes para la cabina española, uno se enfermó y como yo traducía por escrito para una revista teórica Cuestiones actuales del socialismo, estaba al corriente. Ellos ya habían preparado el número dedicado al Congreso y yo había conseguido la ponencia del Presidente, así que cuando se dieron cuenta de que faltaba el otro en la cabina, se les ocurrió contratar al profesor que tradujo el discurso. (Soldatić 2016)

Su máximo alcance como intérprete ha sido la Conferencia General de la UNESCO, la Conferencia General de Interpol y la Conferencia Mundial de la Alimentación FAO, pero en 1985, cuando entró en el servicio diplomático, tuvo que dejar la interpretación simultánea.

La primera traducción literaria que hizo Dalibor Soldatić fue la versión española de una de las más significantes novelas serbias de todos los tiempos, Diccionario jázaro de Milorad Pavić (Anagrama, Barcelona, 1989). Según nos contó el mismo Soldatić, se trataba de una casualidad: "Tuve la suerte de que mi compañero de estudios de México se había trasladado a España y se puso a trabajar para Anagrama y para Carmen Balcells, incluso tuvo una librería en Madrid. El mismo Pavić me ofreció que hiciera la traducción de una novela suya, optamos por el Diccionario jázaro, Jorge Herralde decidió publicarlo tras leer un capítulo y salió una traducción que muchos comentaron que era excelente" (Soldatić 2016). 
En 1993 Soldatić regresó a la Universidad y por primera vez en su vida se ocupó solo de traducciones literarias. Se puso de acuerdo con el director y propietario de la Editorial Laguna para hacer un proyecto muy ambicioso: le propuso que lanzaran una serie de autores españoles modernos: Vázquez Montalbán, Pérez-Reverte, E. Mendoza, Torrente Ballester, R. Sender: "llevaba yo años de frustración de enseñar a los alumnos autores y libros que a veces ellos no podían leer en original, y luego dirigirme a un público lectoral que no sabía nada de eso" (Soldatić, 2016). Varios años después, una casa editorial de Pirot, Pi-press, quiso empezar a publicar autores españoles, y el joven colega de profesor Soldatić, Vladimir Karanović, que se ha especializado en el siglo XIX, les propuso que publicaran una colección de obras de autores de ese siglo, a propósito de hacer que la literatura española esté mucho más presente en el ámbito cultural serbio.

Entre las traducciones de Dalibor Soldatić de la literatura española figuran: Belver Yin de Jesús Ferrero (Laguna, 2006), Crónica del Rey pasmado de Gonzalo Torrente Ballester (Laguna, 2006), La aventura equinoccial de Lope de Aguirre de Ramón J. Sender (Laguna, 2006), Amor, curiosidad, prozac y dudas de Lucía Etxebarría (Laguna, 2007), La caída de Cristóbal Colón. El juicio de Bobadilla de Consuelo Varela (Čigoja štampa, 2008), Señas de identidad de Juan Goytisolo (Laguna, 2009), La desheredada de Benito Pérez Galdós (Pi-press, 2012) y Fortunata y Jacinta de Benito Pérez Galdós (Partenon, 2015). En cuanto a la literatura hispanoamericana, Soldatić tradujo El libro de arena de Jorge Luis Borges (Paideia, 2002) y Crímenes imperceptibles de Guillermo Martínez (Laguna, 2005).

Queremos comentar también el aporte de Dalibor Soldatić a la Antología del cuento hispanoamericano "Večita smrt od ljubavi jača", vol 2 (ed. B. Kovačević Petrović, Tanesi, 2012) con la traducción del cuento "Cuál es la onda" de José Agustín, el relato crucial para la onda mexicana "meksičkom romanu donela potpuno odbacivanje književnih konvencija, uvela je u znatnoj meri erotizam, potpunu slobodu izražavanja, prvo priznanje postepene amerikanizacije meksičkog života, protest protiv tradicionalne političke retorike"22 (Soldatić 2000: 95).

22 "A la novela mexicana la ha traído un rechazo completo de las convenciones literarias, ha introducido el erotismo en la medida significante, igual que la libertad de expresión, el primer reconocimiento de la americanización gradual de la vida mexicana, la protesta en contra de la tradicional política retórica. (Tr. aut.) 


\section{La enseñanza de literatura}

El interés en la literatura hispanoamericana en Serbia también está estrechamente relacionado con el boom. Cuando empezó su internacionalización, la literatura latinoamericana se comercializó mucho, fue un éxito editorial y por consecuencia se puso de moda:

Hay que tener en cuenta que los estudios universitarios son de una $u$ otra manera conservadores. Los estudios académicos no pueden seguir las modas, tienen que guardar distancia para poder ser objetivos. Por lo tanto, la literatura española terminaba con la Generación 27, pero en la literatura hispanoamericana era inevitable tocar la nueva novela porque todo el mundo hablaba de ello y el interés era demasiado marcado. (Soldatić 2016)

En los años setenta y ochenta libros en español podían encontrarse en la librería Mladost en el centro de Belgrado. Al final de cada año académico, el profesor Soldatić les llevaba la lista de lecturas obligatorias y ellos hasta septiembre importaban una cantidad de libros que cubría la necesidad de los estudiantes. Había incluso otras posibilidades: los alumnos también podían pedir lo que les interesaba, y además los libros latinoamericanos los conseguían desde Argentina, de Fernando García Campero, que, según Soldatić, era un librero excepcional. Pero en los años noventa las cosas empeoraron: "Durante los diez años de sanciones, nuestros alumnos han empobrecido y se acostumbraron a fotocopiar libros y otros materiales. Ahora, en el nivel universitario eso está prohibido: el reglamento permite sólo las fotocopias de fragmentos" (Soldatić 2016). Sin embargo, con Internet, la Biblioteca Virtual de Cervantes y libros electrónicos hoy es mucho más fácil conseguir todo lo necesario para los estudios del Hispanismo.

Aunque hay mucho interés en los estudios del Hispanismo en Serbia, según la opinión del profesor Soldatić, a la mayoría de los alumnos hoy en día no les gusta mucho leer, las generaciones actuales están formadas de un modo muy distinto a sus antecesores, siendo a veces muy indiferentes, lo que se debe al desarrollo de la tecnología. El interés por la literatura ha decaído, pero las tiradas de los libros publicados hoy en Serbia son mil ejemplares, como mucho. En la conversación que la autora de 
este artículo tuvo con Dr. Dalibor Soldatić en mayo 2016, él se acordó de dos ejemplos de los periódicos diarios serbios que hicieron campañas con libros clásicos, que se vendían por dos o tres euros en el quiosco y que a las ocho de la mañana ya estaban agotados. Por lo tanto, hay que tener en cuenta la caída del poder adquisitivo de la población. Por otra parte, actualmente, la oferta de la televisión con cien canales durante todo el día es una tentación muy difícil de resistir para ponerse a leer.

La Facultad de Filología de Belgrado en el momento de la creación de este artículo tiene 7.000 estudiantes registrados en la plataforma Moodle, de los 10.000 en total, y de 200 profesores contratados, 150 utiliza la enseñanza electrónica. Según la opinión de Soldatić, es un método muy útil porque en la plataforma se pueden poner los datos generales, y dedicar la clase al análisis, a la esencia. En cuanto a los métodos de enseñanza, Soldatić cree que el futuro está "en la enseñanza electrónica combinada con la enseñanza clásica. Yo tengo mis reservas con la educación a distancia, prefiero el contacto vivo, pero con la enseñanza combinada se abren las posibilidades enormes, tanto para el profesor como para los alumnos" (Soldatić 2016).

\section{Impresiones y conclusiones}

Considerando todos los aspectos del hispanismo en Serbia, se podría decir que Dalibor Soldatić es su piedra de toque y su pilar desde hace más de cuarenta años, y que ha participado y dejado huella en todos sus ámbitos.

En la época de los años sesenta del siglo XX, cuando en Yugoslavia empezó la publicación de las traducciones hechas del español, no había ni diccionarios ni libros adecuados para apoyar a los primeros traductores. Hoy en día, con el progreso de la tecnología, la mayoría de los problemas de traducción se resuelven en unos segundos y casi todo tipo de información se consigue a través de Internet. Toda la política del desarrollo del hispanismo en Serbia ha producido centenas de hispanistas y decenas de buenos traductores, ha subido el nivel de la traducción de los libros hispanos, e influido en el profesionalismo en ese campo tan importante de todas las filologías. 
En los años sesenta, setenta y ochenta, Yugoslavia era uno de los países con un óptimo interés en la literatura hispanoamericana, se traducía y se publicaba mucho más que en Francia, que en aquel entonces todavía no tenía mucho interés en los libros del otro continente, o España, que sufría la dictadura y sus consecuencias. Teniendo en cuenta el tamaño del país y sus recursos económicos, se podría decir que fuimos una superpotencia traductora. Por otro lado, hoy en día, a pesar de tener decenas de buenos traductores, los tirajes son mínimos (entre 300 y 1000 ejemplares) y las remuneraciones que los editores pagan a los traductores humillantes (3-4 euros por página, sin las subvenciones extranjeras) aunque los ejemplos de Politika y Večernje novosti demostraron que existía el público lector, y que el problema yacía en la política de las casas editoriales, porque obviamente el mercado y el interés no faltaba. A cambio del enorme entusiasmo hace medio siglo, y decenas de revistas literarias que publicaban textos y traducciones, actuales y cultos, de óptima calidad, hoy en día la mayoría de las publicaciones semejantes dependen del interés privado de los editores y sus colaboradores.

Durante las cuatro décadas de su docencia en la Facultad de Filología de Belgrado, Dalibor Soldatić enseñaba varias asignaturas, pero se podría decir que su preferido campo de investigación y enseñanza era Literatura Hispanoamericana. A pesar de su jubilación en 2015 de la enseñanza del grado y del puesto de jefe del Departamento de Estudios Ibéricos -donde le sustituyó la actual gerente de la Cátedra, Dra. Jelena Filipović-, Dalibor Soldatić sigue activo en todos los campos hispánicos: en la enseñanza de las asignaturas de maestría -Violencia en la literatura hispanoamericana y La novela hispanoamericana experimental- y de doctorado -Literaturas hispánicas y realidad-, al igual que en la tutoría de las tesis doctorales, artículos y congresos científicos, tertulias y coloquios sobre literatura, ponencias universitarias, edición de revistas hispánicas. Una de sus actuales estudiantes de maestría, Stefana Živojinović, nos comentó sus impresiones sobre la colaboración con su profesor:

El Profesor Soldatić posee un conocimiento impresionante tanto sobre la cultura hispanoamericana como sobre la cultura y la literatura en general, y por lo tanto representa una persona de la que los estudiantes pueden 
aprender mucho. En sus clases nos enseñó a analizar, sentir y amar la literatura hispanoamericana, entender mejor sus visiones y razones, y gracias a él llegamos a sentir esa pasión por la literatura que pocas personas son capaces de transmitir. (Živojinović 2016)

Entre varios colaboradores que Soldatić ha tenido en la Facultad de Filología a lo largo de su carrera y gran apoyo que ha ofrecido a sus jóvenes colegas, secretaria del Departamento de Estudios Ibéricos, Izabela Beljić, destaca otro tipo de contribución de su colega:

De todo lo que he aprendido del profesor Soldatić tal vez lo más valioso fue su empeño en la construcción de las relaciones agradables en nuestro colectivo, llenos de respeto y dignidad en la diversidad de cosas y personas. Gracias a la actitud benévola y amable con todos los colegas, su generosa ayuda en el trabajo y las buenas intenciones en cada colaboración, el profesor Soldatić logró hacer un ambiente de trabajo positivo y agradable. Nuestros esfuerzos de continuar las relaciones profesionales llenas de respeto y amistad en el Departamento de Estudios Ibéricos siguen creciendo, no por una obligación hacia él, sino por nuestro deseo y placer. (Beljić 2016)

Otra aportación del gran hispanista serbio/yugoslavo que hace falta mencionar está relacionada con la Asociación de Hispanistas ${ }^{23}$ serbios, fundada en 2007 y gestionada por Jelena Spasojević, y la actual Red de Hispanistas de Europa Central encabezada por Dra. Ilinca Ilian de la Universitatea de Vest din Timişoara, la asociación que en el encuentro primerizo recibió (a la sugerencia de Soldatić) el nombre de Red Regional de Hispanistas de Hungría, Rumania y Serbia y que le "debe enormemente al sincero, generoso y sabio apoyo del profesor Dalibor Soldatić, que desde su primer encuentro con los miembros de la red, el coloquio de 2009, intitulado Los estudios hispánicos en la región DKTM, mostró su interés por los trabajos conjuntos y las actividades realizadas en equipo" (Ilian 2016), quien militó mucho por el buen funcionamiento de la Red y por la ampliación de las actividades de ésta, y sigue activo como colaborador y miembro en el comité científico de la revista Colindancias. Otra posibilidad rela- 
cionada con la Red es también uno de los proyectos de Dalibor Soldatić, "que esperamos que se realizarán algún día, puesto que hasta ahora no lo conseguimos -creación un máster trilateral dentro de la Red de Hispanistas de Europa Central" (Ilian 2016).

Cuando Dalibor Soldatić vino a la Facultad de Filología de Belgrado tras terminar sus estudios en México, era el único yugoslavo, entre veinte millones de habitantes, con el título de profesor de la Lengua Española y Literaturas Hispánicas; en esa época en el Lectorado Español trabajaba cuatro personas y el Idioma Español fue una asignatura auxiliar. Hoy en Serbia hay más de 1500 hispanistas graduados y alrededor de veinte profesores y lectores empleados en el Departamento de Estudios Ibéricos de la Facultad de Filología de Belgrado, otros tantos en el Departamento la Filología Hispánica de la Facultad de Filología y Artes de Kragujevac y cinco docentes en el Lectorado Español de la Facultad de Filosofía y Letras de Novi Sad. Todos ellos, salvo los lectores extranjeros, fueron estudiantes del profesor Soldatić. A nuestra pregunta cómo ve el futuro del hispanismo en Serbia, Dalibor Soldatić nos respondió:

Primero, creo que inevitablemente se va a superar la actual situación de lejanía de América Latina, con la cual hay en realidad pocos contactos. En el nivel diplomático tenemos solo cuatro embajadas en todo ese continente: en Argentina, México, Cuba y Venezuela, eso también debería cambiar. Creo que se van a intensificar las comunicaciones con España también. Queda absurdo que China y Japón no están lejos, pero América Latina sí lo es. Estoy seguro de que se van a intensificar esos contactos, con lo cual se abrirán las nuevas perspectivas del empleo para los jóvenes hispanistas. (Soldatić 2016)

En el momento cuando más de $\mathbf{4 7 0}$ millones de personas tienen el español como lengua materna y el grupo de usuarios potenciales de español alcanza 560 millones, cuando el español es la segunda lengua materna del mundo por número de hablantes y el 6,7\% de la población mundial es hispanohablante, cuando más de 21 millones de alumnos estudian español como lengua extranjera en diversas regiones del planeta, ese idioma en Serbia se enseña en tres universidades y cen- 
tenas de escuelas públicas y privadas. Sabiendo que el interés por la lengua española en esta región no cesa y que anualmente se publican decenas de traducciones hechas por los hispanistas serbios, estamos seguros que entre todos los profesores, traductores y alumnos habrá buenos herederos de la obra, la docencia y el pensamiento de Dalibor Soldatić, que todavía tienen mucho que aprender de él. Más allá de los hechos aquí plasmados, personalmente me siento muy orgullosa de haber pasado la escuela del profesor Soldatić, segura de que muchos estudiantes y colegas suyos sienten lo mismo.

\section{BIBLIOGRAFÍA}

Anđić, Branko. Comunicación personal, Belgrado, 7 de septiembre de 2015. Entrevista personal grabada.

Anđić, Branko y Dalibor Soldatić. "Panorama nove hispanoameričke književnosti”. Delo, br. 8-9 (1978): 1-3. Štampano.

Bajić, Dragana. Comunicación personal, 1 de junio de 2016. Correo electrónico. Beljić, Izabela. Comunicación personal, 1 de julio 2016. Correo electrónico. Damjanov, Sava. Comunicación personal, 19 de enero 2016. Entrevista personal grabada.

Dickov, Vesna. "Las literaturas hispánicas y el pensamiento crítico y teórico serbio". Colindancias, No. 5 (2014): 75-102. Impreso.

Đurić, Miloš D. "Dalibor Soldatić, Prilozi za teoriju novog hispanoameričkog romana. - Beograd i Kragujevac: Filološki fakultet i Nova Svetlost, 2002 - 290 str.". Filološki pregled, br. XXXII/1 (2005): 175-179. Štampano.

Ilian, Ilinca. Comunicación personal, 16 de julio 2016. Correo electrónico. Josimčević, Marica. "Roman Karlosa Fuentesa Terra Nostra: izazov, svedočanstvo, predskazanje". Sveske, br. X/54-55 (2000): 103-112. Štampano.

Soldatić, Dalibor. "Hispanoamerički roman danas". Delo, br. 8-9 (1978): 28-41. Štampano.

Soldatić, Dalibor. "Karlos Fuentes". Karlos Fuentes. Smrt Artemija Cruza. Zagreb: Zora, 1969. 251-253. Štampano.

Soldatić, Dalibor. "Predgovor Antologiji savremene hispanoameričke pripovetke". Branko Anđić (ur.). Antologija savremene hispanoameričke pripovetke. Beograd: Srpska književna zadruga, 1980. VII-XXVIII. Štampano. 
Soldatić, Dalibor. "Tokovi razvoja meksičke književnosti". Sveske, br. 54-55 (2000): 92-97. Štampano.

Soldatić, Dalibor. Prilozi za teoriju novog hispanoameričkog romana. Beograd, Kragujevac : Filološki fakultet, Nova svetlost, 2002. Štampano.

Soldatić, Dalibor. "Pedro Paramo - povest o neuspešnom traganju". Beogradski književni časopis, br. 2/15 (2006): 215-219. http://www. bgknjizevnicasopis.rs/preuzimanje/tekstovi/broj02.pdf (3. 7. 2016).

Soldatić, Dalibor i Željko Donić. Svet hispanistike. Beograd: Zavod za udžbenike, 2011). Štampano.

Soldatić, Dalibor. Comunicación personal, Budva, 20 de junio de 2015. Entrevista personal grabada.

Soldatić, Dalibor. Comunicación personal, Novi Sad, 12 de mayo de 2016. Entrevista personal grabada.

Živojinović, Stefana. Comunicación personal, 16 de julio 2016. Correo electrónico.

\title{
Bojana Kovačević Petrović
}

\section{DALIBOR SOLDATIĆ AND HISPANIC AMERICAN LITERATURE IN SERBIA}

\begin{abstract}
Summary
Through various interviews and statements made for the purpose of this research, this article aims to show the contribution of Dr. Dalibor Soldatic to Hispanic studies in Yugoslavia and Serbia, as one of the pioneers of Hispanism in this region, honored for his work with the Medal of the Order of Isabella the Catholic, granted by the Government of Spain, and the Medal of the Mexican Order of the Aztec Eagle. Considering his career as a professor at the Faculty of Philology of the University of Belgrade and Head of the Department of Iberian Studies at the same institution, his scientific articles published in various academic journals, his translation of twenty books, his experience as Vice Dean of Education and International Relations and Vice Dean of Science and International Relations at the Faculty of Philology (two terms) and Vice Chancellor of International Relations at the University of Belgrade, as well as his support for students and younger colleagues, in this paper we shall focus on Dr. Soldatićs impact on the reception of Spanish American literature in Serbia and his
\end{abstract}


critical thought about Hispanic issues. A part of our article will be dedicated to the teaching of Spanish and Latin American literature in the 45 years of Hispanism in this region and Soldatićs vision of future studies of Hispanic philology.

Keywords: Hispanism in Yugoslavia, Hispanic studies in Serbia, translation from Spanish into Serbian, Faculty of Philology of the University of Belgrade, reception of Latin American literature in Serbia, teaching of Spanish and Latin American literature. 